\title{
Validating Blackberry Seedling Pedigrees and Developing an Improved Multiplexed Microsatellite Fingerprinting Set
}

\author{
Jason D. Zurn \\ USDA-ARS National Clonal Germplasm Repository, Corvallis, OR
}

\author{
Katie A. Carter \\ Department of Horticulture, Oregon State University, Corvallis, OR \\ Melinda H. Yin, Margaret Worthington, and John R. Clark \\ Department of Horticulture, University of Arkansas, Fayetteville, AR
}

Chad E. Finn

USDA-ARS Horticultural Crops Research Unit, Corvallis, OR

\author{
Nahla Bassil ${ }^{1}$ \\ USDA-ARS National Clonal Germplasm Repository, Corvallis, OR
}

\begin{abstract}
AdDitional INDEX words. Rubus, RosBREED, mixed-ploidy populations, phylogeny, Plant Variety Protection, plant identification, pedigree confirmation

Abstract. Confirming parentage and clonal identity is an important aspect of breeding and managing germplasm collections of clonally propagated, outcrossing crops, like blackberry (Rubus subgenus Rubus). DNA fingerprinting sets are used to identify off-cross progeny and confirm clonal identity. Previously, a six-simple sequence repeat (6SSR) fingerprinting set was developed for blackberry using a small number of samples. The usefulness of the 6-SSR fingerprinting set for pedigree confirmation had not been evaluated. Therefore, it was used in this study to validate parentage for 6 and 12 biparental populations from the University of Arkansas (UA) and US Department of Agriculture Agricultural Research Service (USDA-ARS), Horticultural Crops Research Unit (HCRU) breeding programs, respectively. Twenty-seven of the 489 individuals in these breeding populations were identified as off-cross. The 6-SSR fingerprinting set was sufficient for parentage confirmation; however, a total of 61 plants distributed across 28 sets of genotypes could not be distinguished from each other. An 8-SSR fingerprinting set with improved resolution was subsequently developed and used to evaluate 177 Rubus accessions from the USDA-ARS National Clonal Germplasm Repository, UA, and USDA-ARS HCRU programs. The 8-SSR fingerprinting set distinguished all samples expected to have unique genotypes and identified differing DNA fingerprints for two sets of accessions suspected to have identical fingerprints. Cluster analysis grouped the accessions from the eastern and western US breeding programs based on geography and descent. Future work will focus on establishing a database of DNA fingerprints for germplasm identification and for determining pedigree relationships between blackberry accessions.
\end{abstract}

Blackberry is one of the many important commercialized fruit crops within the Rosaceae. The aggregate fruit produced by these plants have been enjoyed worldwide by people throughout history (Clark and Finn, 2011; Hummer and Janick, 2007). In North America, the majority of cultivars are polyploid, but the Rubus genus has a range of ploidy levels ranging from diploid to dodecaploid (Clark and Finn, 2011; Meng and Finn, 2002; Thompson, 1995). These cultivars often have complicated pedigrees consisting of many species from the genus. Except a few regionally important species, nearly all

\footnotetext{
Received for publication 13 June 2018. Accepted for publication 9 Aug. 2018. The authors would like to thank April Nyberg, Jill Bushakra, Harrison Schreiber, Mary Peterson, Melissa Clark, Jaime Willard, David Gilmore, Tralena Kay Buck, Dan Chapman, and staff at the University of Arkansas System Division of Agriculture Fruit Research Station for their technical support. This research was funded through the USDA National Institute of Food and Agriculture Specialty Crop Research Initiative project "RosBREED: Combining Disease Resistance and Horticultural Quality in New Rosaceous Cultivars" (2014-51181-22378) and by the USDA-ARS CRIS projects 2072-21000-04900D and 2072-21220-003-00D.

${ }^{1}$ Corresponding author. E-mail: nahla.bassil@ars.usda.gov.
}

cultivated blackberries contain two or more Rubus species from four (Allegheniensis, Arguti, Rubus, and Ursini) of the 12 identified sections of the subgenus Rubus in their pedigrees (Clark and Finn, 2011; Watson, 1958). Clark et al. (2007) classified North American blackberry cultivars into two groups. The first group consists of erect and semierect blackberries $(2 n=4 x=28)$ and trailing dewberries $(2 n=2 x=14)$ domesticated from diploid and tetraploid species from eastern North America. The second group is comprised of trailing blackberries generated from polyploid species from western North America, such as Rubus ursinus (ploidy ranging from $8 x$ to $12 x$ ), and introgressions of tetraploid blackberry and diploid red raspberry ( $R$. idaeus, Idaeobatus) through intersectional hybrids [e.g., 'Logan' $(2 n=6 x=42)$, 'Tayberry' $(2 n=6 x=$ $42)$, 'Boysenberry' $(2 n=7 x=49)$, and 'Youngberry' $(2 n=7 x=$ 49)]. Trailing cultivars are generally of higher ploidy than the tetraploid erect eastern North American cultivars and can be found with ploidy levels ranging from $6 x$ to $10 x$ and $12 x$, in addition to occasional aneuploids (Meng and Finn, 2002; Thompson, 1995).

The identification of cultivars and validation of pedigree is an important aspect of blackberry breeding and maintaining 
a germplasm collection as cultivars are clonally propagated and hybridize readily with nearby plants (Clark and Finn, 2011). DNA fingerprinting sets have become a useful tool to identify cultivars, validate pedigrees, study population diversity, and protect breeders' rights (Laurentin, 2009; Peace, 2017). Before the use of DNA-based techniques, cultivar identification, pedigree validation, and population diversity for many plants were assessed by comparing morphology (Gilbert et al., 1999; Laurentin, 2009). Morphological traits can be highly influenced by environmental conditions, and closely related individuals may appear phenotypically the same (Gilbert et al., 1999; Laurentin, 2009). Of existing DNA-based markers, simple sequence repeat (SSR) markers are the preferred markers for cultivar identification and parentage confirmation. SSRs are abundant in plant genomes, cost-effective to use, amenable to automated scoring with software, and can be amplified in multiplex PCR (Bassil et al., 2016; Peace, 2017; Weising et al., 2005). Moreover, SSRs are highly polymorphic even amongst full-siblings and can be used to identify parental and sibling relationships (Bassil et al., 2012). In Rubus, SSRs were first isolated from red raspberry (Graham et al., 2002, 2004, 2006), and shown to cross-amplify in blackberry (Bassil et al., 2010; Bradish et al., 2016; Stafne et al., 2005). Before 2008, a small number of SSRs were isolated from blackberry species including eight SSRs from $R$. alceifolius (Amsellem et al., 2000), 15 from $R$. hochstetterorum (Lopes et al., 2006), and eight from 'Marion' blackberry (Castillo et al., 2010). Lewers et al. (2008) generated expressed sequence tags (ESTs) from 'Merton Thornless' blackberry and predicted the presence of 940 polymorphic SSRs in the genome based on evaluating 'APF-12' and 'Arapaho' with 33 SSRs. Thousands of SSRs were also recently identified from transcriptome sequencing of 'Loch Ness' blackberry (Garcia-Seco et al., 2015). SSRs used for cultivar identification in blackberry predominately had dinucleotide motifs (Bassil et al., 2016; Castillo et al., 2010; Marulanda et al., 2007, 2012). These dinucleotide motif SSRs can exhibit various artifacts such as stutters, split peaks, and binning errors (Bassil et al., 2016; Pompanon et al., 2005;
Weising et al., 2005). SSRs with nucleotide motifs greater than two often do not exhibit these artifacts (Weising et al., 2005) and are recommended for use in fingerprinting sets (Akin et al., 2016; Testolin and Cipriani, 2010).

Bassil et al. (2016) recently evaluated 15 trinucleotidecontaining SSRs in 13 diverse blackberry cultivars and created a 6-SSR fingerprinting set that distinguished these cultivars. The Bassil et al. (2016) 6-SSR fingerprinting set had not been assessed for its ability to confirm parentage and the resolution of the set was unknown due to the small number of samples evaluated in the previous study. The objective of this study was to evaluate this 6-SSR fingerprinting set for its ability to confirm parentage and distinguish seedlings of breeding populations from the University of Arkansas and the USDA-ARS Horticultural Crops Research Unit breeding programs; to develop an improved fingerprinting set, if needed; and to evaluate the new set for germplasm identification in a subset of the USDA-ARS National Clonal Germplasm Repository (NCGR) blackberry collection.

\section{Materials and Methods}

Germplasm. Crosses were made in 2011, 2012, and 2013 to create 6 and 12 biparental populations at the UA and the HCRU programs, respectively (Table 1; Supplemental Table 1). These 520 individuals were evaluated with the 6-SSR fingerprinting set. The biparental populations and the parental germplasm from the UA were grown at the UA Fruit Research Station (Clarksville, AR). The seedlings and parental germplasm in the HCRU program were grown at Oregon State University's North Willamette Research and Extension Center (Aurora, OR) and at the Lewis-Brown Farm (Corvallis, OR). Additionally, 71 Rubus subgenus Rubus accessions from the NCGR, HCRU, and UA and a subset of 106 individuals evaluated with the 6SSR fingerprinting set were evaluated with the newly developed 8-SSR fingerprinting set (Supplemental Table 1).

DNA Extraction. Young actively growing leaf tissue from the apex of primocanes was collected from the populations and

Table 1. Summary of the blackberry populations from the University of Arkansas (UA) and the USDA-ARS Horticultural Crops Research Unit (HCRU) breeding programs.

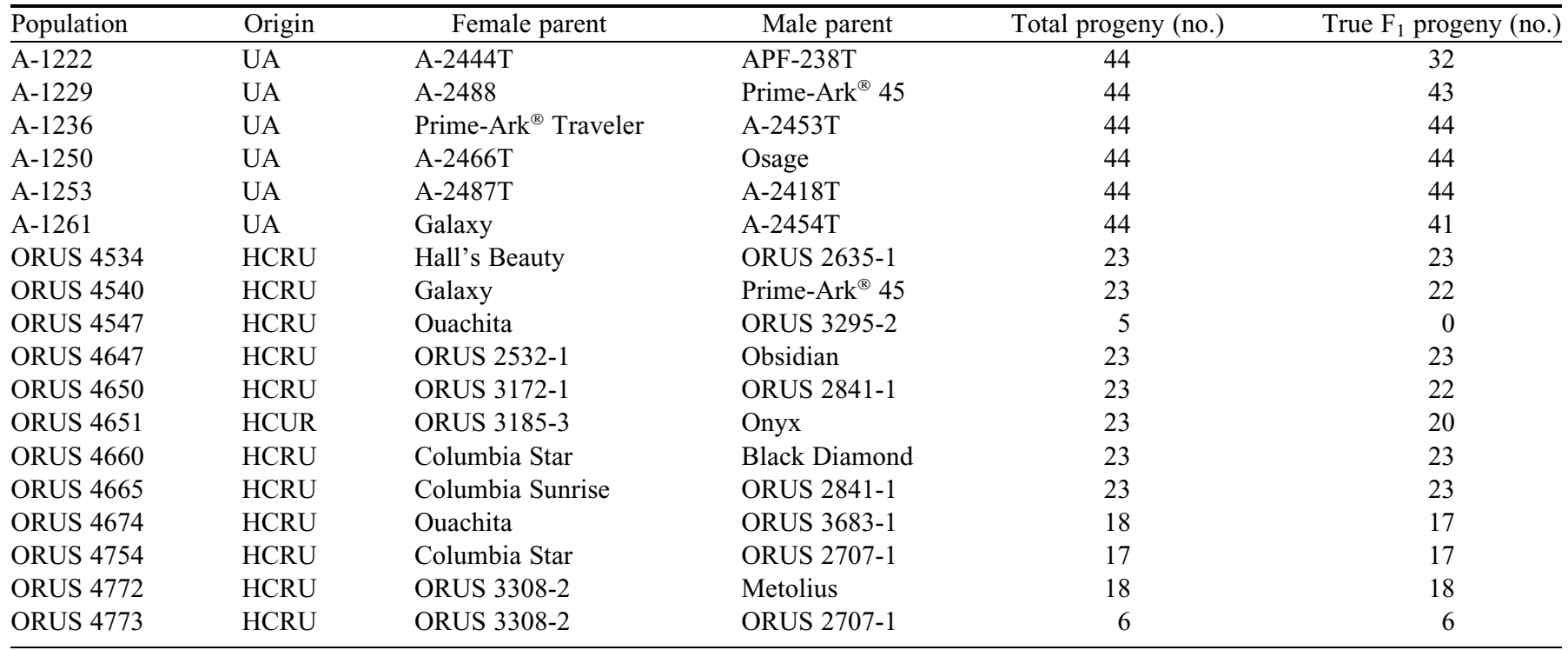


their parents. In the event that individuals did not have actively growing primocanes, the youngest possible leaf material was harvested. For DNA extraction, 30-50 mg of tissue were arrayed into two 96-well plates either containing silica to desiccate the samples for UA samples or steel beads for ORgrown samples from the HCRU or NCGR collections. The UA samples were shipped overnight to the NCGR. After desiccating for 1 week, the samples were ground using a mixer mill (MM 301; Retsch International, Hann, Germany). For ORgrown samples, the samples were frozen in liquid nitrogen and kept at $-80{ }^{\circ} \mathrm{C}$ until ready for extraction. Oregon-grown samples were ground with a mixer mill (MM 301) and kept frozen by immersing in liquid nitrogen before grinding and halfway through the grinding. For all samples, DNA was extracted using a modified DNA extraction protocol [Puregene; Qiagen, Hilden, Germany (Gilmore et al., 2011)]. DNA was quantified via spectrophotometry using a multilabel plate reader (Victor3V 1420; PerkinElmer, Waltham, MA) and diluted to $3 \mathrm{ng} \cdot \mu \mathrm{L}^{-1}$.

Pedigree validation USING The 6-SSR Fingerprinting SET. The populations were evaluated with the 6-SSR fingerprinting developed by Bassil et al. (2016) (Table 2). Polymerase chain reaction (PCR) was conducted using a microsatellite PCR kit (Type-it, Qiagen) in $15-\mu \mathrm{L}$ reactions consisting of $8.3 \mu \mathrm{L}$ of $2 \times$ Type-it Multiplex PCR Master Mix (Qiagen), 1.5 $\mu \mathrm{L}$ of PCR grade water, $1.7 \mu \mathrm{L}$ of multiplexed primer mix, and $3.5 \mu \mathrm{L}$ of DNA at a concentration of $3 \mathrm{ng} \cdot \mu \mathrm{L}^{-1}$. The final concentration for each of the primers was $0.014 \mu \mathrm{M}$ for RH_MEa0013dA06, $0.020 \mu \mathrm{M}$ for RH_MEa0006bG05, $0.021 \mu \mathrm{M}$ for RH_MEa0007aG06, $0.085 \mu \mathrm{M}$ for RH_MEa0011dG03a and RH_MEa0016bC11, and 0.113 $\mu \mathrm{M}$ for ERubLR_SQ07-4_D05. Amplification was performed in a thermal cycler (PTC-225; MJ Research, Waltham, MA) using a program consisting of an initial denaturation at $95^{\circ} \mathrm{C}$ for $5 \mathrm{~min} ; 10$ cycles of denaturation at $95{ }^{\circ} \mathrm{C}$ for $30 \mathrm{~s}$, annealing for $1.5 \mathrm{~min}$ starting at $65^{\circ} \mathrm{C}$ and decreasing by $1{ }^{\circ} \mathrm{C}$ per cycle, and extension at $72^{\circ} \mathrm{C}$ for $30 \mathrm{~s} ; 30$ cycles of denaturation at $95^{\circ} \mathrm{C}$ for $30 \mathrm{~s}$, annealing at $55^{\circ} \mathrm{C}$ for $1.5 \mathrm{~min}$, and extension at $72^{\circ} \mathrm{C}$ for $30 \mathrm{~s}$; followed by a $30 \mathrm{~min}$ hold at $60{ }^{\circ} \mathrm{C}$. Following completion of the program, the amplicons remained at $4{ }^{\circ} \mathrm{C}$ until removed from the thermocycler. Reaction success was assessed via $2 \%$ agarose gel electrophoresis and successful reactions were further evaluated with a capillary electrophoresis system (CEQ 8000; Beckman Coulter, Brea, CA). The fragment analysis module of CEQ 8000 software was used for allele visualization and sizing, with individuals scored for presence or absence of an allele by grouping alleles into bins of less than one base pair (bp). Pedigree confirmation was conducted by identifying alleles in progeny and comparing to alleles in both parents. If the suspected offspring's DNA fingerprint had an allele that was not found in either parent, the individual was labeled as "offcross." Suspected offspring with only alleles that could be found in one or both parents were labeled as "true $\mathrm{F}_{1} \mathrm{~s}$."

Creation of an improved 8-SSR Fingerprinting SET. Fifteen additional SSRs were evaluated to improve the Bassil et al. (2016) 6-SSR fingerprinting set (Table 2). An M13 labeling method (Schuelke, 2000) was used to initially evaluate these SSRs on progeny that could not be differentiated using the 6-SSR fingerprinting set and the parents of the populations. Reactions were conducted in $15-\mu \mathrm{L}$ volumes. Similar to the previously described reactions, the M13 PCRs consisted of $8.3 \mu \mathrm{L}$ of $2 \times$ Type-it Multiplex PCR Master Mix (Qiagen),
1.5 $\mu \mathrm{L}$ of PCR grade water, $1.7 \mu \mathrm{L}$ of primer mix, and $3.5 \mu \mathrm{L}$ of DNA at a concentration of $3 \mathrm{ng} \cdot \mu \mathrm{L}^{-1}$. The primers were evaluated at a final concentration of $0.057 \mu \mathrm{M}$ for the forward primer and $0.227 \mu \mathrm{M}$ for the reverse and M13 primers. Thermocycling conditions were as previously described; however, the annealing temperature during the 10 touchdown cycles began at $62{ }^{\circ} \mathrm{C}$ decreasing by $1{ }^{\circ} \mathrm{C}$ per cycle to a temperature of $52{ }^{\circ} \mathrm{C}$ and the second annealing temperature was $52^{\circ} \mathrm{C}$. Upon successful amplification, PCR products were pooled and alleles were separated via capillary electrophoresis (CEQ 8000). The markers were evaluated for their ease of scoring, lack of artifacts, the ability to distinguish unique accessions, and allelic size range. WellRED-labeled (Beckman Coulter) forward primers were ordered from Integrated DNA Technologies (Coralville, IA) for the best performing SSRs and primer concentrations were optimized for the conditions described for the 6-SSR fingerprinting set.

COMPARISON OF THE 6-SSR AND 8-SSR FINGERPRINTING SETS. The 6-SSR and 8-SSR fingerprinting sets were compared with one another on a subset of 106 individuals that had been evaluated with both fingerprinting sets. For each marker, the number of observed alleles and observed genotypes were counted and Nei's gene diversity (Nei, 1978) was calculated at the allelic and genotypic level using the "locus_table" command in "Poppr" (Kamvar et al., 2014, 2015). The mean alleles and genotypes per locus and mean Nei's gene diversity were also calculated for each fingerprinting set.

ASSESSMENT OF POPUlation STRUCTURE FOR THE 8-SSR FINGERPRINTING SETS. Population structure was evaluated for the 8-SSR fingerprinting set by constructing a dendrogram using the R packages "ape" [version 5.0 (Paradis et al., 2004)] and "Poppr" [version 2.6.1 (Kamvar et al., 2014, 2015)]. Null alleles were coded as "NA" and unknown alleles (e.g., masking of null alleles or unknown dosage configurations) were coded as "000" using the "recode_polyploids" command (Supplemental Table 2) (Bruvo et al., 2004; Kamvar et al., 2014, 2015). The "bruvo.boot" command (Kamvar et al., 2014, 2015) was used to produce a neighbor joining tree with the "njs" algorithm from "ape" and Bruvo's distance (Bruvo et al., 2004) assuming an infinite allele, allele-sharing model (Supplemental Table 2) (Metzger et al., 2016). Bootstrap support of 2000 permutations was used (Supplemental Table 2). Bruvo's distance was chosen for its ability to calculate distances for mixed-ploidy populations (Bruvo et al., 2004). K-means clustering was also used to visualize the data. When identifying the number of clusters for K-means clusters, the maximum number of principal components were retained and the number of clusters were selected based on the lowest Bayesian information criterion value.

\section{Results}

Pedigree validation using the 6-SSR fingerprinting Set. The 6-SSR fingerprinting set was able to confirm parentage for the majority of the offspring (Table 1; Supplemental Table 1). Of the 489 progeny, 462 individuals $(94.5 \%)$ were found to be true $F_{1}$ s. Three of the six UA populations and five of the 12 HCRU populations had off-cross progeny (Table 1). All five individuals from the HCRU population ORUS 4547 were offcross with the male parent being unknown. All progeny could be distinguished from one another for the UA population A1222 and the HCRU populations ORUS 4547, ORUS 4647, 


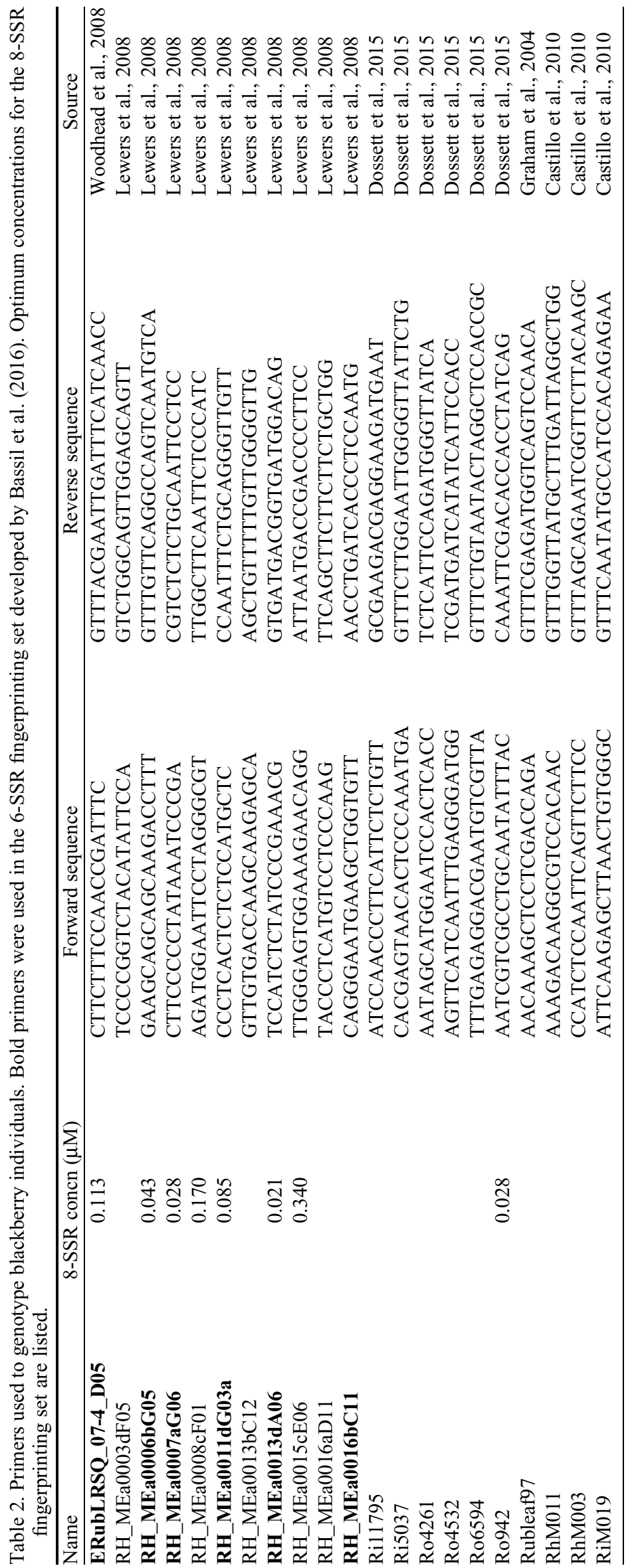


ORUS 4660, ORUS 4674, ORUS 4754, ORUS 4772, and ORUS 4773. In the remaining populations, a total of 61 individuals (49 from UA and 12 from HCRU) were distributed across 28 groups of individuals with identical fingerprints. The individuals that could not be differentiated consisted mostly of progeny; however, the parents A-2488 and 'Prime-Ark ${ }^{\circledR}$ Traveler' were found to have identical fingerprints to the $\mathrm{F}_{1} \mathrm{~s} A$ 1229-15 and A-1236-51, respectively.

Creation OF AN IMPROVEd 8-SSR FINGERPRINTING SET. The 6-SSR fingerprinting set was improved upon to create an 8-SSR fingerprinting set by evaluating the 61 undifferentiated samples and population parents with 15 new SSRs (Table 2). The SSRs RH_MEa003dF05, RH_MEa0013bC12, Ri5037, Ro4261, Ro4532, Ro6594, and RiM019 were not able to differentiate any of the individuals with identical fingerprints. Ro942 and RH_MEa0015cE06 amplified well, were easy to score, and were highly polymorphic. Of the 28 groups of individuals with identical fingerprints, 10 groups could be distinguished using Ro942 and 11 groups could be distinguished with RH_MEa0015cE06. The allele ranges for these primer pairs ranged from 100 to $180 \mathrm{bp}$ for Ro942 and 225 to $275 \mathrm{bp}$ for RH_MEa0015cE06. The ranges of these primers could be accommodated through the use of WellRed dyes, which were not used for other primers in these ranges in the 6-SSR set. RH_MEa0008cF01 was able to further differentiate one group which could not be differentiated by either Ro942 or RH_MEa0015cE06. The allelic range of RH_MEa0008cF01 was also between 100 and $180 \mathrm{bp}$. The upper range of Ro942 and RH_MEa0008cF01 overlapped slightly with the lower range of RH_MEa0016bC11; however, both R0942 and RH_MEa0008cF01 were more polymorphic. Therefore, RH_MEa0016bC11 was removed to avoid confusion when identifying alleles. After RH_MEa0016bC11 was removed, a non-specific 200-bp amplicon was not detected in subsequent multiplex reactions. This non-specific amplicon appeared to be the result of an off-target amplification with RH_MEa0007aG06 or RH_MEa0011dG03a. In conjunction, RH_MEa0008cF01, RH_MEa0015cE06, and Ro942 were able to resolve all but 10 sets of two individuals. One more set of individuals, ORUS 4540U and ORUS 4540T, could be further differentiated using the SSRs RiM019 or Rubleaf97. Unfortunately, the allelic ranges of these markers prevented them from being integrated into a single multiplexed reaction as all dye combinations were already in use. WellRed-labeled forward primers were ordered for RH_MEa0008cF01,
RH_MEa0015cE06, and Ro942. The primer concentrations for the 8-SSR assay were optimized for $15 \mu \mathrm{L}$ reactions with identical components and thermocycling conditions to 6-SSR fingerprinting set (Table 2; Fig. 1) (Bassil et al., 2016).

COMPARISON OF THE 6-SSR AND 8-SSR FINGERPRINTING SETS. A subset of 106 samples that were genotyped with both fingerprinting sets, consisting of 63 individuals from the UA breeding program and 43 individuals from the USDA-ARS HCRU breeding program, was used to compare the 6-SSR and 8-SSR fingerprinting sets (Supplemental Table 1). The average number of alleles, average number of genotypes, and Nei's 1978 gene diversity was higher for the 8-SSR fingerprinting set than the 6-SSR fingerprinting set (Table 3) (Nei, 1978). RH_MEa0016bC11 had the second lowest gene diversity when calculated based on the alleles and the third lowest when calculated based on genotypes (Table 3). As such, RH_MEa0016bC11 was removed to make the 8-SSR fingerprinting set. ERubLRSQ_07-4_D05 performed the worst of all of the SSRs tested, with only three observed alleles, and was not polymorphic in the UA breeding program germplasm (Table 3 ). ERubLRSQ_07-4_D05 is a good candidate for replacement in the set. More work is needed to identify a suitable replacement within the 225 to 275-bp allelic range of ERubLRSQ_074_D05. The 8-SSR fingerprinting set performed better than the 6-SSR fingerprinting set when evaluating the two fingerprinting sets on subsets of germplasm from the UA and HCRU breeding programs (Table 3). A greater number of alleles and higher allelic and genotypic diversity parameters were observed in the HCRU subset than in the UA subset.

EVALUATION OF THE 8-SSR FINGERPRINTING SET IN DIVERSE GERMPLASM. A total of 177 accessions were evaluated with the 8-SSR fingerprinting set, consisting of 79 accessions from UA and other eastern North American breeding programs, 66 accessions from the HCRU breeding program, four accessions from Europe, one accession from Brazil, and 27 Rubus subgenus Rubus species, most of which are preserved at the NCGR (Supplemental Table 1). Within the species germplasm, 14 accessions originated from eastern North America, eight originated from western North America, and the remaining five originated from Spain, Russia, Pakistan, Turkey, and Montenegro. Six accessions ('Columbia Star', 'Galaxy', 'Kiowa', 'Loch Ness', 'Marion', and 'Prime Ark ${ }^{\circledR}$ 45') from different sources (UA, HCRU, or NCGR) were found to have identical fingerprints (Fig. 2). 'Thornless Evergreen' and 'Everthornless' were found to have the same fingerprint. This
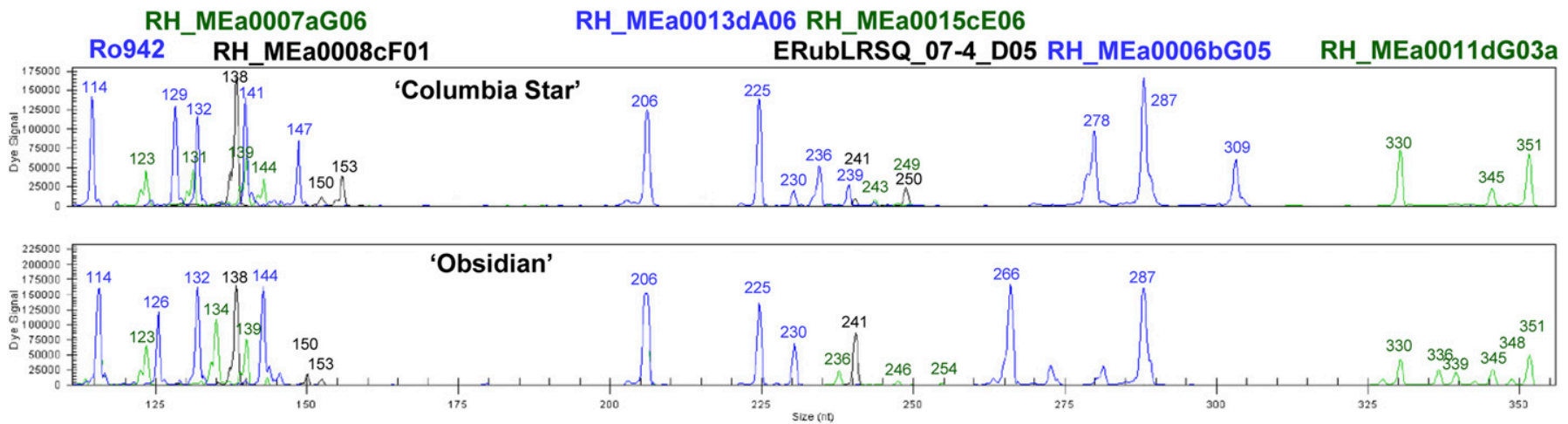

Fig. 1. Electropherograms for the 8-SSR fingerprinting set for the blackberries 'Columbia Star' and 'Obsidian'. 
Table 3. Summary statistics for each marker in the 6-SSR and 8-SSR fingerprinting (FP) sets on a subset of 106 blackberry accessions genotyped with both fingerprinting sets. Summary statistics for subsets consisting of 43 individuals from the USDA-ARS Horticultural Crops Research Unit (HCRU) breeding program and 63 individuals from the University of Arkansas (UA) breeding program are also shown.

\begin{tabular}{|c|c|c|c|c|c|}
\hline FP set & Locus & $\begin{array}{c}\text { Observed } \\
\text { alleles (no.) }\end{array}$ & $\begin{array}{l}\text { Nei’s } 1978 \text { gene } \\
\text { diversity (allelic) }\end{array}$ & $\begin{array}{c}\text { Observed } \\
\text { genotypes (no.) }\end{array}$ & $\begin{array}{c}\text { Nei’s } 1978 \text { gene } \\
\text { diversity (genotypic) }\end{array}$ \\
\hline \multicolumn{6}{|c|}{ All samples } \\
\hline 6-SSR & RH_MEa0016bC11 & 10 & 0.72 & 18 & 0.82 \\
\hline Both & RH_MEa0013dA06 & 11 & 0.84 & 38 & 0.94 \\
\hline Both & ERubLRSQ_07-4_D05 & 3 & 0.44 & 5 & 0.44 \\
\hline Both & RH_MEa0006bG05 & 10 & 0.81 & 27 & 0.80 \\
\hline 8-SSR & Ro942 & 16 & 0.86 & 41 & 0.94 \\
\hline 8-SSR & RH_MEa0008cF01 & 9 & 0.83 & 34 & 0.96 \\
\hline \multirow[t]{3}{*}{ 8-SSR } & RH_MEa0015cE06 & 12 & 0.84 & 40 & 0.95 \\
\hline & Mean 6-SSR & 9.33 & 0.76 & 27.17 & 0.82 \\
\hline & Mean 8-SSR & 10.38 & 0.79 & 32.50 & 0.87 \\
\hline \multicolumn{6}{|c|}{ UA breeding program } \\
\hline Both & RH_MEa0006bG05 & 4 & 0.50 & 7 & 0.56 \\
\hline Both & RH_MEa0011dG03a & 5 & 0.74 & 9 & 0.86 \\
\hline 8-SSR & Ro942 & 9 & 0.73 & 14 & 0.86 \\
\hline 8-SSR & RH_MEa0008cF01 & 6 & 0.81 & 20 & 0.94 \\
\hline \multirow{4}{*}{ 8-SSR } & RH_MEa0015cE06 & 7 & 0.77 & 20 & 0.91 \\
\hline & Mean 6-SSR & 4.67 & 0.57 & 9.17 & 0.64 \\
\hline & Mean 8-SSR & 5.75 & 0.64 & 12.75 & 0.74 \\
\hline & \multicolumn{5}{|c|}{ USDA-ARS HCRU breeding program } \\
\hline 6-SSR & RH_MEa0016bC11 & 10 & 0.77 & 16 & 0.88 \\
\hline Both & RH_MEa0007aG06 & 12 & 0.90 & 33 & 0.99 \\
\hline Both & RH_MEa0013dA06 & 11 & 0.88 & 32 & 0.99 \\
\hline & Mean 8-SSR & 10.13 & 0.85 & 23.50 & 0.94 \\
\hline
\end{tabular}

is not unexpected as 'Everthornless' was derived by isolating the thornless epidermal layer from the periclinal chimera 'Thornless Evergreen' using tissue culture (McPheeters and Skirvin, 2000). Interestingly, two accessions of 'Burbank Thornless' (CRUB 250.001 and CRUB 1815.001) from different sources had different fingerprints but grouped together in the dendrogram. Moreover 'Young Thornless' (CRUB 2003.001) was substantially different from 'Young' (CRUB 131.001). As 'Young Thornless' is reported to be a thornless sport of 'Young' they would be expected to have identical fingerprints (Coyner et al., 2005). All remaining accessions, except for the 10 groups of siblings that could not be differentiated, were found to have different fingerprints. When counting the observed number of alleles, the observed genotypes, and calculating Nei's 1978 genetic diversity, all of the SSRs performed well with the exception of ERubLRSQ_074_D05 (Table 4; Nei, 1978). ERubLRSQ_07-4_D05 continued to exhibit lower allelic and genotypic diversity than the other
SSRs in the 8-SSR fingerprinting set when evaluating a more diverse set of germplasm.

Hierarchical clustering using a Bruvo's distance neighborjoining tree identified distinct groups that separated primarily according to geography and pedigree (Fig. 2). In general, these six groups include a clade of western North American germplasm; a clade of eastern North American species germplasm; a clade of cultivars with 'Merton Thornless' in their pedigree; a group of clades containing Rubus species not native to North America; and two groups of multiple clades containing germplasm that originates from or has a parent from the UA breeding program (Fig. 2). Only one accession, 'Columbia Giant' (CRUB 2694.001) originating from western North America and a full-sib relative of 'Columbia Star', grouped unexpectedly with eastern North American germplasm (Fig. 2). This was only observed using hierarchical clustering and not with $\mathrm{K}$ means clustering. Geographical separation was also observed when conducting K-means clustering that identified eight 


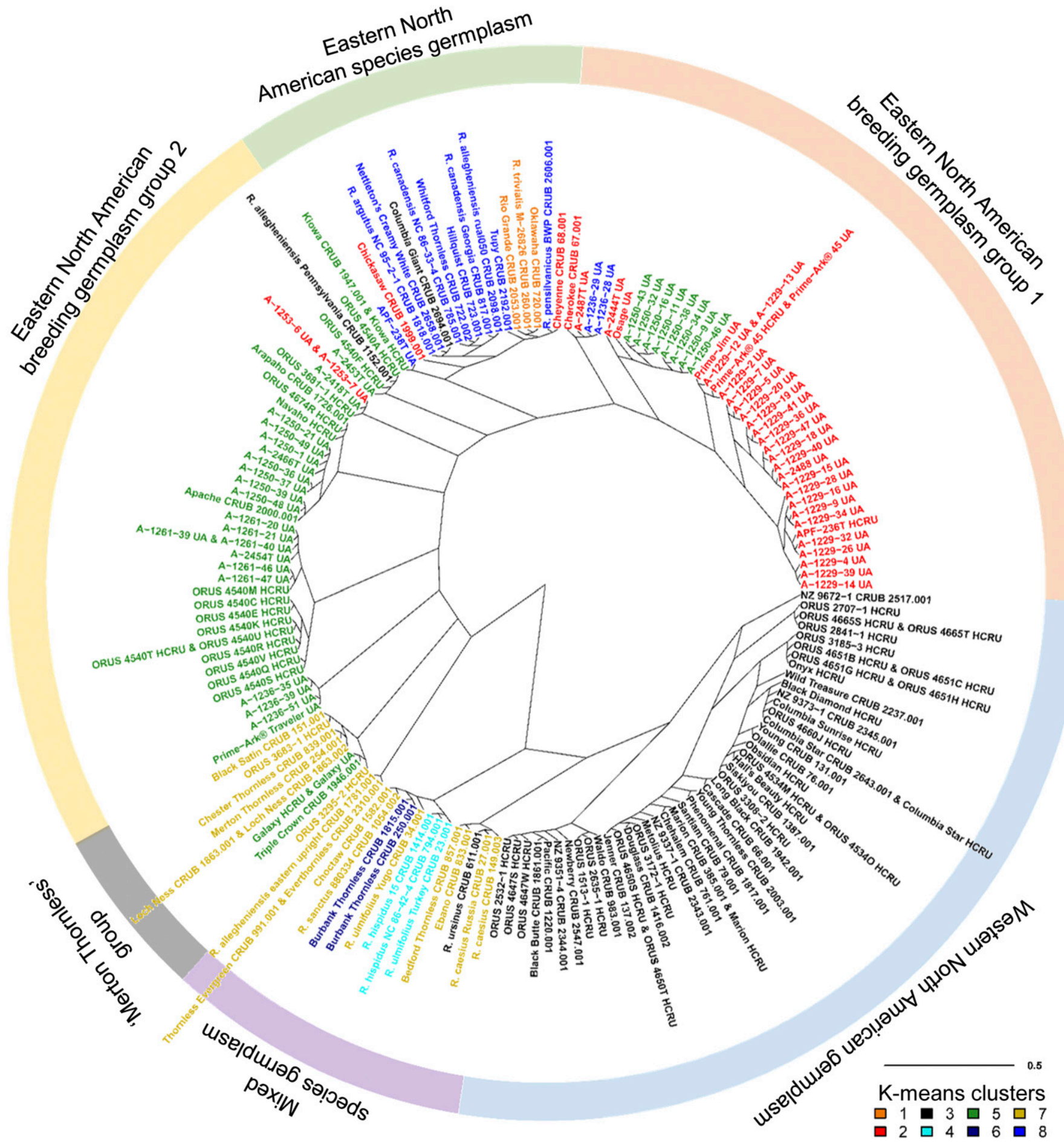

Fig. 2. Radial dendrogram of 177 blackberry accessions evaluated with the 8-SSR fingerprinting set. K-means clustering results for each accession are displayed as different colors for each group. Six sub-groups based on hierarchical clustering are denoted. K-means clustering identified groupings based on geography and descent.

clusters after retaining 120 principal components (Fig. 2). Cluster 3 contained germplasm from western North American breeding programs and was distinct from the eastern North American germplasm. More structure was observed within the eastern North American germplasm and multiple clusters existed (Fig. 2). Clusters 2 and 5 consisted mostly of eastern North American hybrid Rubus cultivars and progeny from biparental crosses where one parent originated from the UA breeding program. Clusters 1,4 , and 8 consisted primarily of species germplasm that originated from the eastern United States. Many of the cultivars found in these clusters had $R$. trivialis or $R$. riograndis in their pedigree. The two 'Burbank Thornless' accessions comprised cluster 6. Finally, cluster 7 was comprised primarily of species germplasm from various geographical locations and 'Merton Thornless' and its descendants (Fig. 2). Nine of the 17 accessions within cluster 7 
Table 4. Summary statistics for each marker in the 8-SSR fingerprinting set on the 177 blackberry accessions genotyped with 8-SSR fingerprinting set.

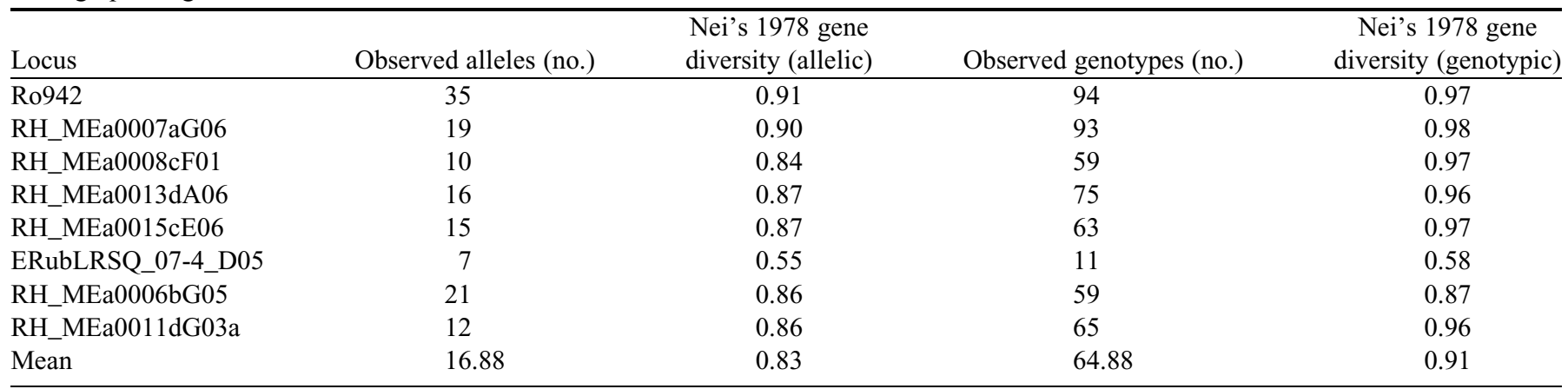

originated from outside of North America (Supplemental Table 1) and an additional five ('Black Satin' CRUB 151.001, 'Chester Thornless' CRUB 839.001, 'Ebano' CRUB 833.001, ORUS 3295-2, and ORUS 3683-1) accessions have European heritage through 'Merton Thornless' derivatives in their ancestry.

\section{Discussion}

The 6-SSR fingerprinting set (Bassil et al., 2016) was found to be sufficient for determining parentage for the UA and HCRU biparental populations (Table 1). The resolution of the 6-SSR fingerprinting set was lacking and individuals from 10 populations had identical DNA fingerprints. Most of the sets of identical individuals were found in the UA populations. The UA breeding program breeds exclusively tetraploid cultivars. Conversely, the ploidy of cultivars produced by HCRU program is known to range from tetraploid to decaploid with the majority thought to be hexaploid (Meng and Finn, 2002; Thompson, 1995). Higher ploidy would allow for more potential gamete combinations during meiosis from each parent resulting in progeny that can be distinguished. The newly developed 8-SSR fingerprinting set was able to differentiate many of the individuals with identical DNA fingerprints. The remaining 10 groups of two undifferentiated individuals consisted of individuals that were planted adjacently to one another in the field. It is likely that the same plant may have been sampled twice as plants could have intergrown in the field.

None of the true $\mathrm{F}_{1}$ samples re-genotyped with the 8-SSR fingerprinting set were found to be off-cross. The new markers included in the 8-SSR fingerprinting set were able to distinguish all but 10 of the 28 groups of individuals that could not be distinguished with the 6-SSR fingerprinting set. Of these 10 groups, only one (ORUS 4540U and ORUS 4540T) could be further distinguished using the SSRs RiM019 and Rubleaf 97 that could not be incorporated into the fingerprinting set. Therefore, the 6-SSR set efficiently confirmed parentage and the 8-SSR set resulted in enhanced distinguishing ability over that provided by the 6-SSR set as illustrated by the higher diversity parameters (Table 3 ). This improved fingerprinting set also distinguished homonyms and synonyms and will be useful for efficient management of the blackberry collection and elimination of synonyms. As expected based on higher ploidy (Meng and Finn, 2002; Thompson, 1995), a greater number of alleles and genotypes and higher diversity estimates were observed in the HCRU subset than in the UA subset. ERubLRSQ_07-4_D05 had substantially lower genetic diversity than the other SSRs in the 8-SSR fingerprinting set and is a candidate for replacement during any future improvements. The lower genetic diversity of ERubLRSQ_07-4_D05 was not surprising given it was designed from a red raspberry EST (Woodhead et al., 2008). SSRs created from ESTs are often conserved across closely related species; however, they display lower polymorphism when compared with non-genic SSRs (Chabane et al., 2005; Varshney et al., 2005).

The varying ploidy levels of hybrid blackberries and the frequency with which these levels change can pose unique challenges when comparing samples using traditional phylogenetic approaches. The majority of distance calculations were devised for diploid organisms and do not account for varying ploidy or allelic dosage (Bruvo et al., 2004). To address these problems, Bruvo et al. (2004) developed a distance calculation that factored in microsatellite mutations assuming a slippedstrand mispairing model and genome additions and losses. The genome addition and loss components of Bruvo's distance were not as applicable to hybrid blackberries as genomes are gained or lost from one generation to the next rather than over the course of thousands of years. Moreover, in its original form Bruvo's distance could only accommodate SSR markers. A solution to this problem was proposed by Metzger et al. (2016) by setting the repeat length parameter of Bruvo's distance to a value smaller than one nucleotide. By doing so, the model becomes an allele-sharing model similar to Prevosti's absolute genetic distance while still accounting for varying ploidy levels (Metzger et al., 2016; Prevosti et al., 1975). The approach proposed by Metzger et al. (2016) can be further extended to experiments using single nucleotide polymorphisms by coding the nucleotides as 1, 2, 3, and 4, making it a valuable tool for studying the relationships of mixed-ploidy populations.

$\mathrm{K}$-means clustering tended to group individuals by identityby-descent and hierarchical clustering grouped individuals by identity-by-state (Fig. 2). This is exemplified by 'Columbia Giant' (CRUB 2694.001) grouping with eastern North American germplasm rather than with western North American germplasm when using hierarchical clustering but not K-means clustering (Fig. 2). The genotypes of the two accessions of 'Burbank Thornless' (CRUB 250.001 and CRUB 1815.001) were different for three of the eight markers evaluated. Interestingly, a common allele was observed for each of the differing SSRs for the two accessions of the diploid 'Burbank Thornless' (Thompson, 1995). As half of the alleles were shared, it is possible that one of the accessions is an offspring of the other or both accessions are half-sibling offspring of 'Burbank Thornless'. The fingerprints of 'Young Thornless' 
(CRUB 2003.001) and 'Young' (CRUB 131.001) were substantially different at each locus tested. 'Young Thornless' was donated by a private grower in Washington State. It is likely that one of these accessions was misidentified. In the cases of 'Burbank Thornless' and 'Young', further work with additional collections of the respective accessions will be needed to fully resolve these differences and identify a true-to-type candidate. The dendrogram and K-means cluster show the HCRU program is genetically distinct from the UA breeding program and other eastern North American germplasm. The accessions in the species groups clustered based on geography. The clusters observed also fit very well to the growth habit groupings established by Clark et al. (2007). This finding was not as readily apparent when using hierarchical clustering and was more discernible when using K-means clustering. As such, this manuscript serves as an excellent example of the importance of analyzing SSR data with multiple methods to clearly identify links between accessions.

The 8-SSR fingerprinting set is an excellent tool for parentage and identity confirmation, as well as plant variety protection. Future work will focus on evaluating additional accessions within the NCGR blackberry germplasm collection to establish a large working library to aid in accession identification and diversity preservation. Additional work is needed to identify the correct accessions of 'Burbank Thornless', 'Young', and 'Young Thornless' and to assess the 8-SSR fingerprinting set's performance in other Rubus subgenera.

\section{Literature Cited}

Akin, M., A. Nyberg, J. Postman, S. Mehlenbacher, and N.V. Bassil. 2016. A multiplexed microsatellite fingerprinting set for hazelnut cultivar identification. Eur. J. Hort. Sci. 81:327-338.

Amsellem, L., J.L. Noyer, T. Le Bourgeois, and M. Hossaert-McKey. 2000. Comparison of genetic diversity of the invasive weed Rubus alceifolius Poir. (Rosaceae) in its native range and in areas of introduction, using amplified fragment length polymorphism (AFLP) markers. Mol. Ecol. 9:443-455.

Bassil, N.V., M. Muminova, and W. Njuguna. 2010. Microsatellitebased fingerprinting of western blackberries from plants, IQF berries and puree. Acta Hort. 859:73-80.

Bassil, N.V., A.M. Nyberg, C.E. Finn, J.R. Clark, C.P. Peace, and A. Iezzoni. 2016. Development of a multiplexed fingerprinting set in blackberry. Acta Hort. 1133:89-96.

Bassil, N.V., A. Nyberg, K.E. Hummer, J. Graham, M. Dossett, and C. E. Finn. 2012. A universal fingerprinting set for red raspberry. Acta Hort. 946:83-88.

Bradish, C.M., E. Overbaugh, J. Ballington, G.E. Fernandez, and N.V. Bassil. 2016. Comparative diversity analysis of southeastern Rubus germplasm through molecular and pedigree techniques. Acta Hort. 1127:157-161.

Bruvo, R., N.K. Michiels, T.G. D'Souza, and H. Schulenburg. 2004. A simple method for the calculation of microsatellite genotype distances irrespective of ploidy level. Mol. Ecol. 13:2101-2106.

Castillo, N.R.F., B.M. Reed, J. Graham, F. Fernández-Fernández, and N.V. Bassil. 2010. Microsatellite markers for raspberry and blackberry. J. Amer. Soc. Hort. Sci. 135:271-278.

Chabane, K., G.A. Ablett, G.M. Cordeiro, J. Valkoun, and R.J. Henry. 2005. EST versus genomic derived microsatellite markers for genotyping wild and cultivated barley. Genet. Resources Crop Evol. 52:903-909.

Clark, J.R. and C.E. Finn. 2011. Blackberry breeding and genetics. Fruit Veg. Cereal Sci. Biotechnol. 5:27-43.

Clark, J.R., E.T. Stafne, H.K. Hall, and C.E. Finn. 2007. Blackberry breeding and genetics. Plant Breed. Rev. 29:19-144.
Coyner, M.A., R.M. Skirvin, M.A. Norton, and A.G. Otterbacher. 2005. Thornlessness in blackberries. Small Fruits Rev. 4:83-106.

Dossett, M., J.M. Bushakra, B. Gilmore, C.A. Koch, C. Kempler, C.E. Finn, and N.V. Bassil. 2015. Development and transferability of black and red raspberry microsatellite markers from short-read sequences. J. Amer. Soc. Hort. Sci. 140:243-252.

Garcia-Seco, D., Y. Zhang, F.J. Gutierrez-Mañero, C. Martin, and B. Ramos-Solano. 2015. RNA-Seq analysis and transcriptome assembly for blackberry (Rubus sp. var. Lochness) fruit. BMC Genomics 16:5.

Gilbert, J.E., R.V. Lewis, M.J. Wilkinson, and P.D.S. Caligari. 1999. Developing an appropriate strategy to assess genetic variability in plant germplasm collections. Theor. Appl. Genet. 98:1125-1131.

Gilmore, B.S., N.V. Bassil, and K.E. Hummer. 2011. DNA extraction protocols from dormant buds of twelve woody plant genera. J. Amer. Pomol. Soc. 65:201-207.

Graham, J., K. Smith, K. MacKenzie, L. Jorgenson, C. Hackett, and W. Powell. 2004. The construction of a genetic linkage map of red raspberry (Rubus idaeus subsp. idaeus) based on AFLPs, genomicSSR and EST-SSR markers. Theor. Appl. Genet. 109:740-749.

Graham, J., K. Smith, I. Tierney, K. MacKenzie, and C.A. Hackett. 2006. Mapping gene $H$ controlling cane pubescence in raspberry and its association with resistance to cane botrytis and spur blight, rust and cane spot. Theor. Appl. Genet. 112:818-831.

Graham, J., K. Smith, M. Woodhead, and J. Russell. 2002. Development and use of simple sequence repeat SSR markers in Rubus species. Mol. Ecol. Notes 2:250-252.

Hummer, K.E. and J. Janick. 2007. Rubus iconography: Antiquity to the renaissance. Acta Hort. 759:89-106.

Kamvar, Z.N., J.C. Brooks, and N.J. Grünwald. 2015. Novel R tools for analysis of genome-wide population genetic data with emphasis on clonality. Front. Genet. 6:208.

Kamvar, Z.N., J.F. Tabima, and N.J. Grünwald. 2014. Poppr: An R package for genetic analysis of populations with clonal partially clonal, and/or sexual reproduction. PeerJ 2:e281.

Laurentin, H. 2009. Data analysis for molecular characterization of plant genetic resources. Genet. Resources Crop Evol. 56:277-292.

Lewers, K.S., C.A. Saski, B.J. Cuthbertson, D.C. Henry, M.E. Staton, D.S. Main, A.L. Dhanaraj, L.J. Rowland, and J.P. Tomkins. 2008. A blackberry (Rubus L.) expressed sequence tag library for the development of simple sequence repeat markers. BMC Plant Biol. 8:69.

Lopes, M.S., G. Belo Maciel, D. Mendonça, F. Sabino Gil, and A. Da Câmara Machado. 2006. Isolation and characterization of simple sequence repeat loci in Rubus hochstetterorum and their use in other species from the Rosaceae family. Mol. Ecol. Resour. 6:750-752.

Marulanda, M.L., A.M. López, and S.B. Aguilar. 2007. Genetic diversity of wild and cultivated Rubus species in Columbia using AFLP and SSR markers. Crop Breed. Appl. Biotechnol. 7:242-252.

Marulanda, M., A.M. López, and M. Uribe. 2012. Molecular characterization of the Andean blackberry, Rubus glaucus, using SSR markers. Genet. Mol. Res. 11:322-331.

McPheeters, K. and R.M. Skirvin. 2000. 'Everthornless' blackberry. HortScience 35:778-779.

Meng, R. and C.E. Finn. 2002. Determining ploidy level and nuclear DNA content in Rubus by flow cytometry. J. Amer. Soc. Hort. Sci. 127:767-775.

Metzger, M.J., A. Villalba, M.J. Carballal, D. Iglesias, J. Sherry, C. Reinisch, A.F. Muttray, S.A. Baldwin, and S.P. Goff. 2016. Widespread transmission of independent cancer lineages within multiple bivalve species. Nature 534:705-709.

Nei, M. 1978. Estimation of average heterozygosity and genetic distance from a small number of individuals. Genetics 89:583-590.

Paradis, E., J. Claude, and K. Strimmer. 2004. APE: Analyses of phylogenetics and evolution in R language. Bioinformatics 20:289290.

Peace, C.P. 2017. DNA-informed breeding of rosaceous crops: Promises, progress and prospects. Hort. Res. 4:17006. 
Pompanon, F., A. Bonin, E. Bellemain, and P. Taberlet. 2005. Genotyping errors: Causes, consequences and solutions. Nat. Rev. Genet. 6:847-859.

Prevosti, A., J. Ocaña, and G. Alonso. 1975. Distances between populations of Drosophila subobscura, based on chromosome arrangement frequencies. Theor. Appl. Genet. 45:231-241.

Schuelke, M. 2000. An economic method for the fluorescent labeling of PCR fragments. Nat. Biotechnol. 18:233-234.

Stafne, E.T., J.R. Clark, C.A. Weber, J. Graham, and K.S. Lewers. 2005. Simple sequence repeat (SSR) markers for genetic mapping of raspberry and blackberry. J. Amer. Soc. Hort. Sci. 130:722728.

Testolin, R. and G. Cipriani. 2010. Molecular markers for germplasm identification and characterization. Acta Hort. 859:59-72.
Thompson, M.M. 1995. Chromosome number of Rubus cultivars at the national clonal germplasm repository. HortScience 30:1453-1456.

Varshney, R.K., A. Graner, and M.E. Sorrells. 2005. Genic microsatellite markers in plants: Features and applications. Trends Biotechnol. 23:48-55.

Watson, C.W.R. 1958. Handbook of the Rubi of Great Britain and Ireland. Vol 11. Cambridge Univ. Press, Cambridge, UK.

Weising, K., H. Nybom, K. Wolff, and G. Kahl. 2005. DNA fingerprinting in plants: Principles, methods, and applications. CRC Press, New York, NY.

Woodhead, M., S. McCallum, K. Smith, L. Cardle, L. Mazzitelli, and J. Graham. 2008. Identification, characterisation and mapping of simple sequence repeat (SSR) markers from raspberry root and bud ESTs. Mol. Breed. 22:555-563. 
Supplemental Table 1. List of all blackberry accessions evaluated, the source of the accession, which fingerprinting set the accession was genotyped with, and the results of the parentage analysis for the six University of Arkansas (UA) populations and 12 USDA-ARS Horticultural Crops Research Unit (HCRU) populations. The accessions tested were collected from the UA, HCRU, or the USDA-ARS National Clonal Germplasm Repository (NCGR). The results of the parentage testing are also displayed for progeny tested with the 6-SSR fingerprinting set. Progeny listed as "true $\mathrm{F}_{1} \mathrm{~s}$ " were identified to likely be the result of a cross between the suspected parents. Those listed as "off-cross" had at least one allele that could not be found in either of the suspected parents.

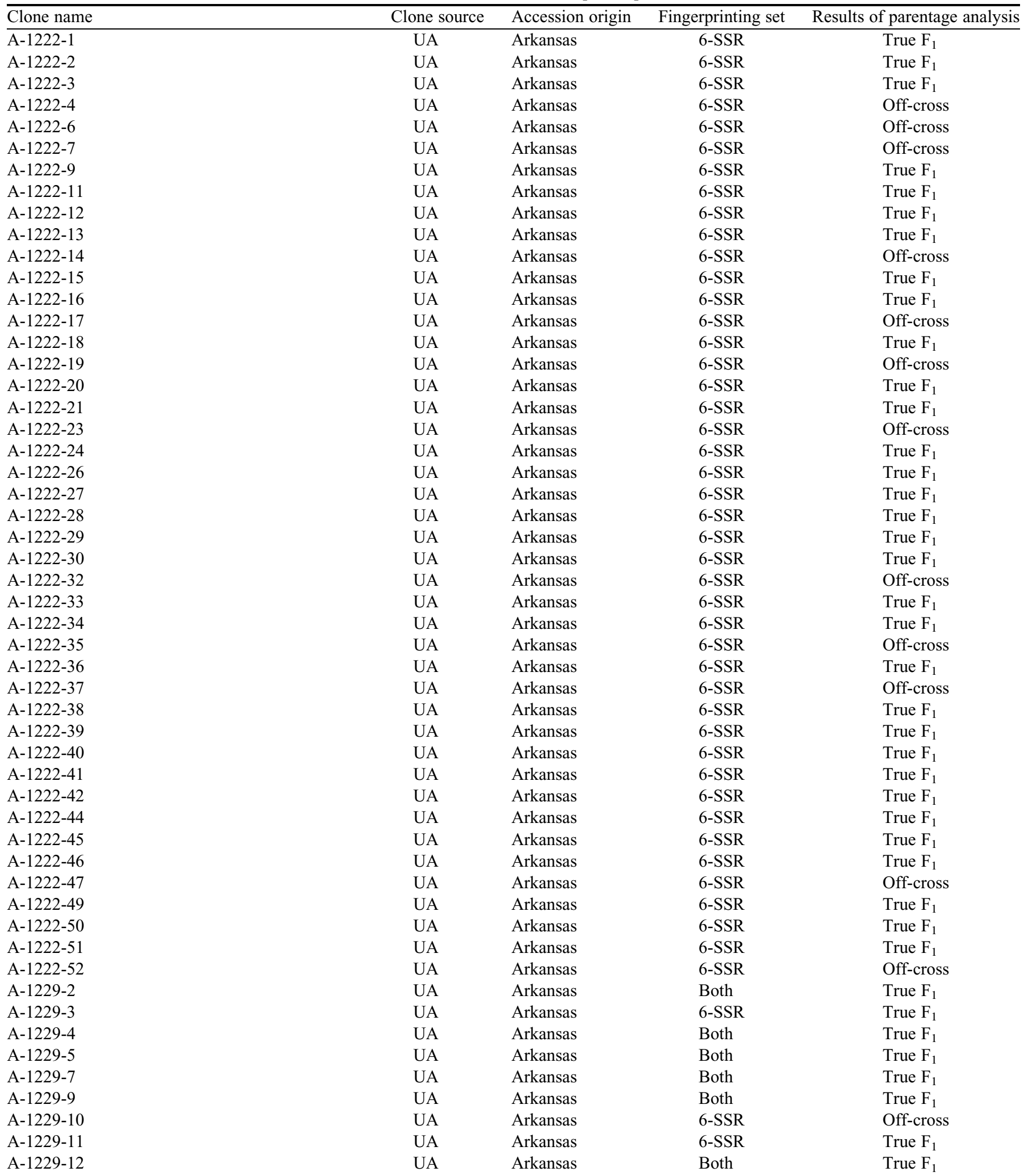


Supplemental Table 1. Continued.

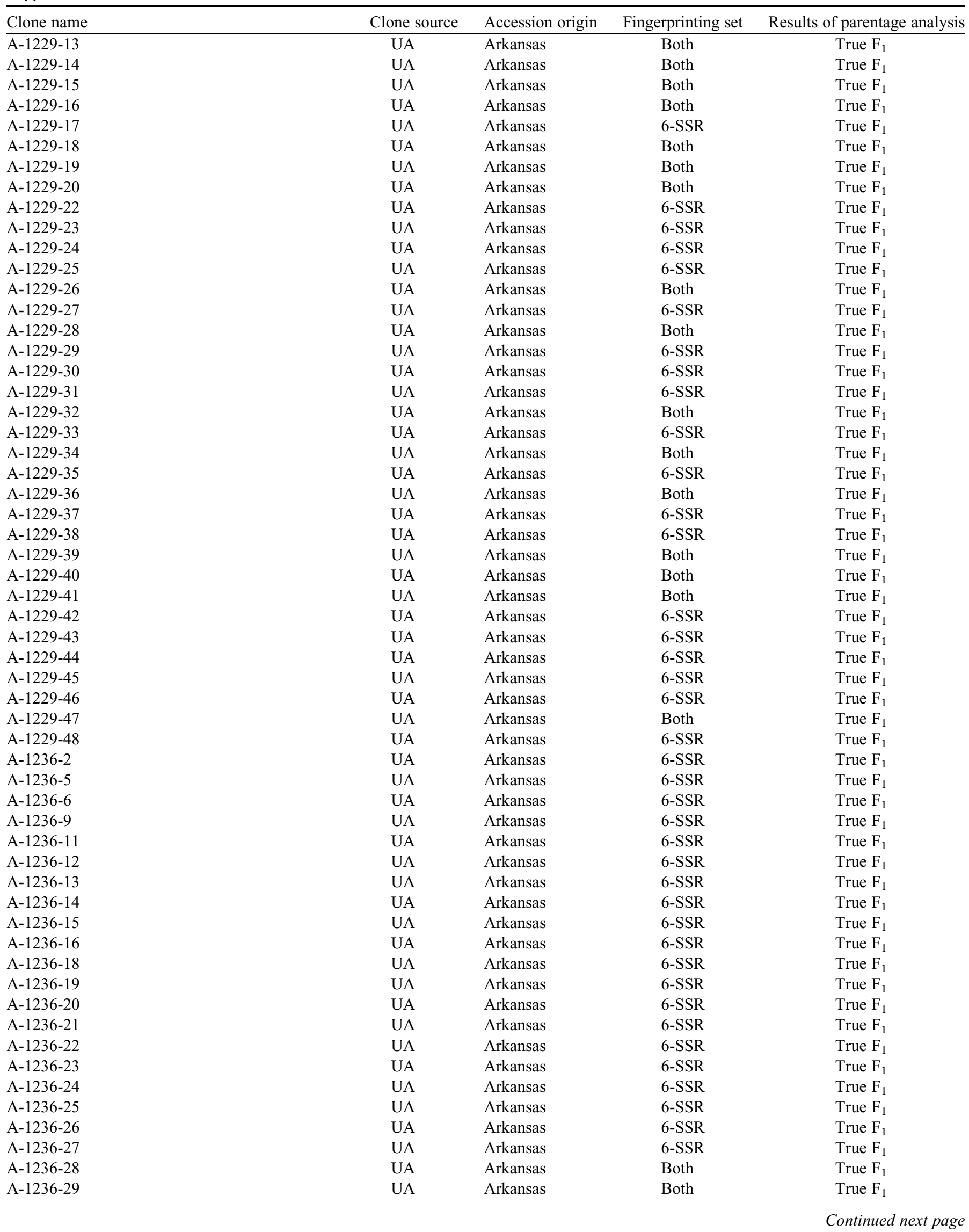


Supplemental Table 1. Continued.

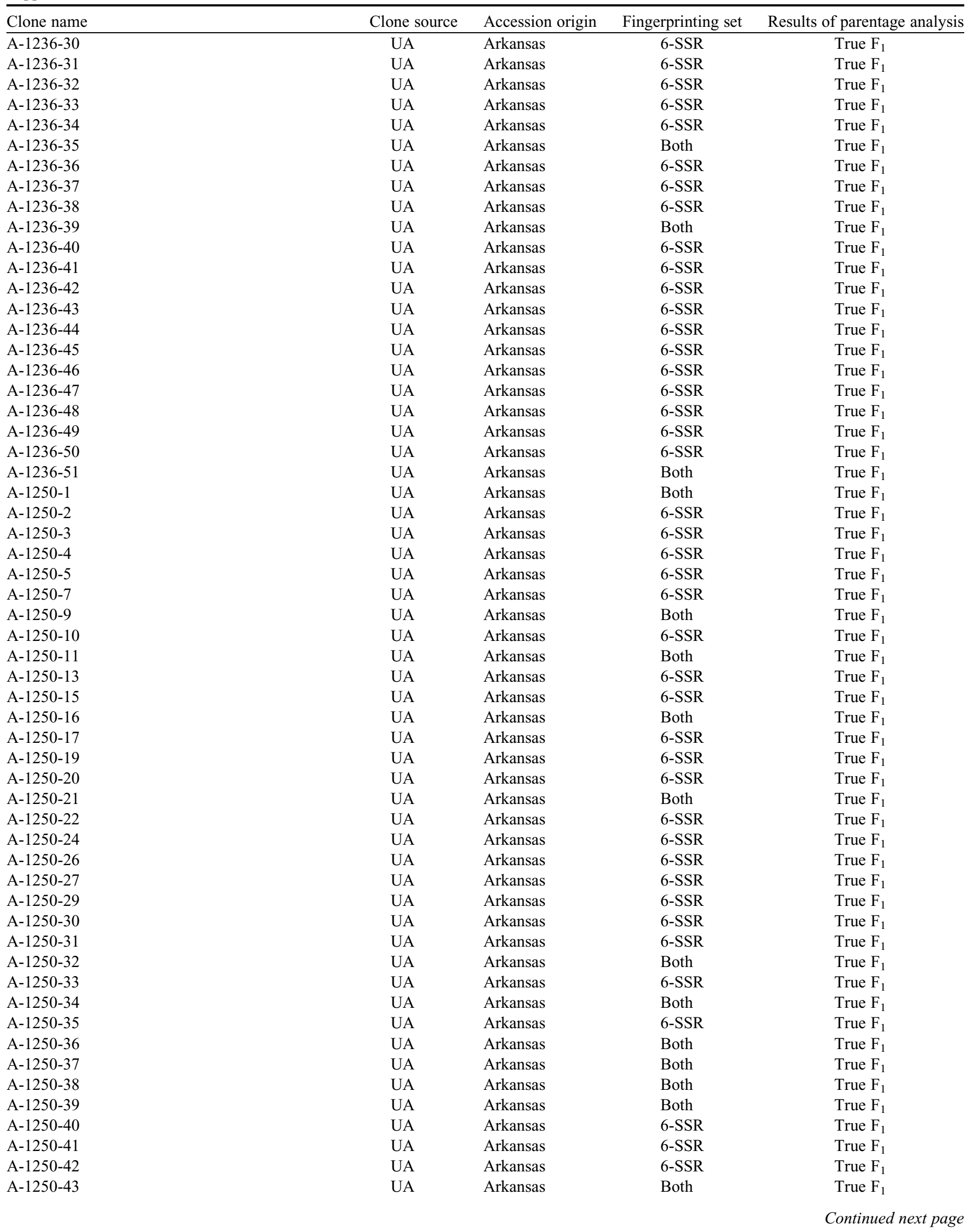


Supplemental Table 1. Continued.

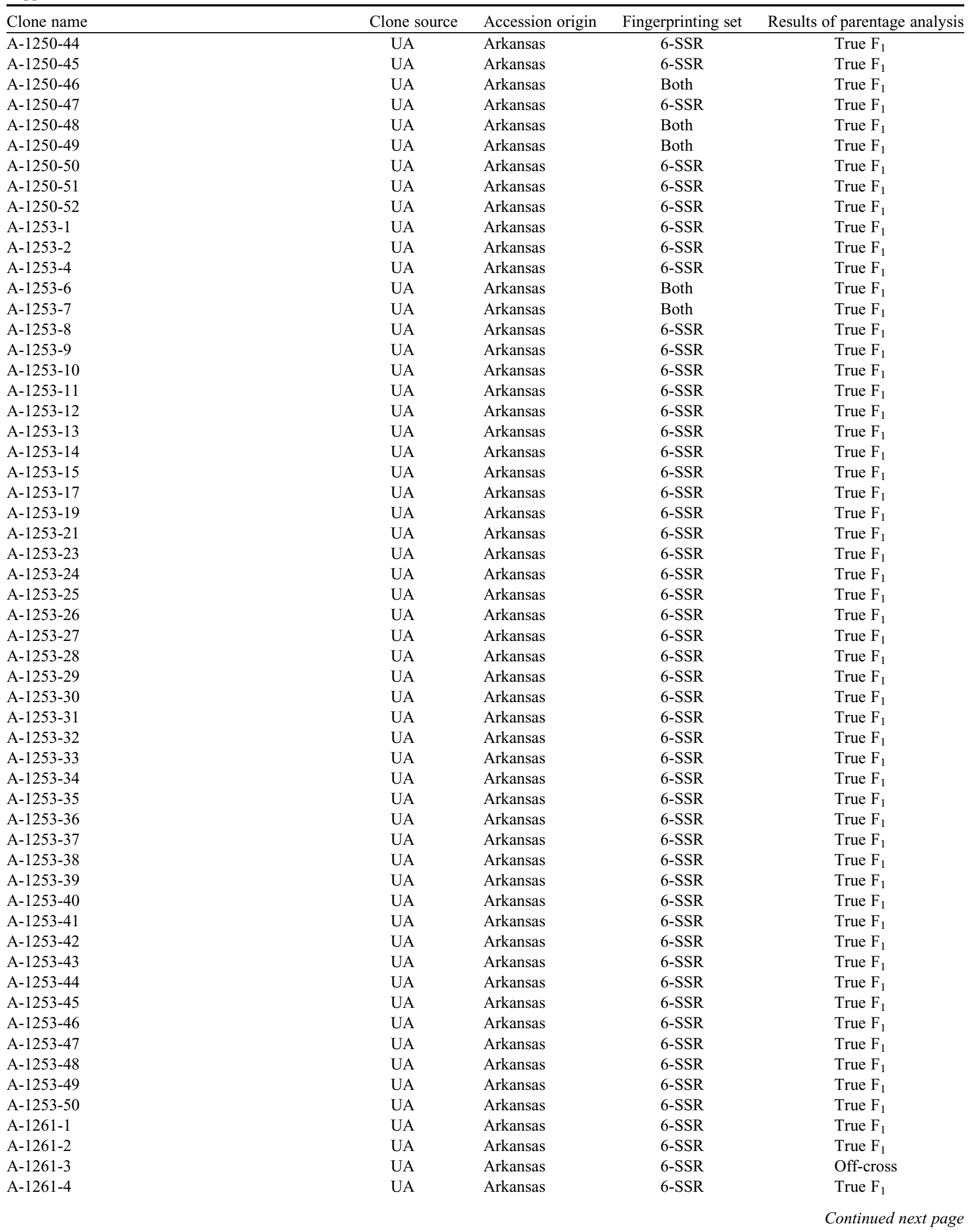


Supplemental Table 1. Continued.

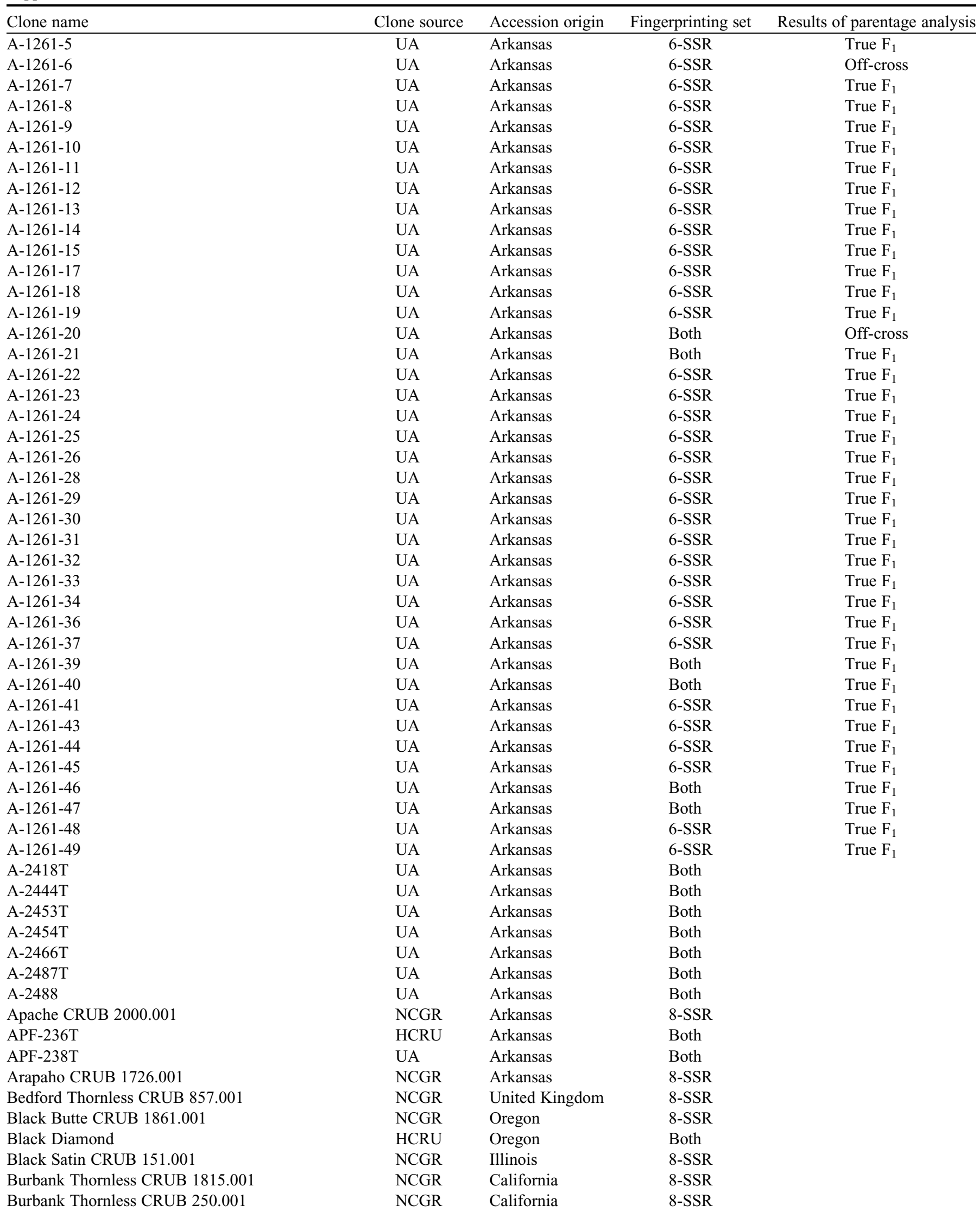


Supplemental Table 1. Continued.

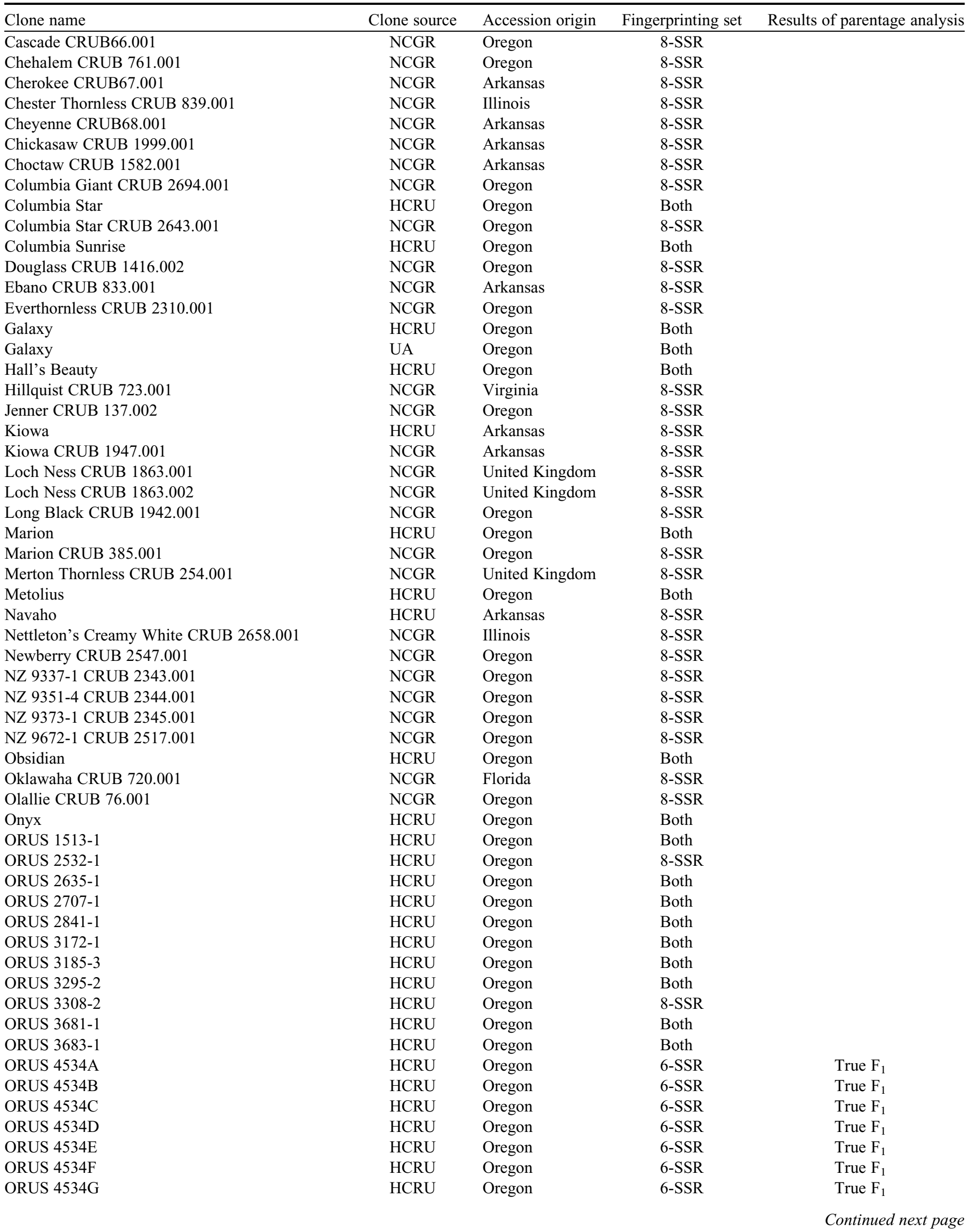


Supplemental Table 1. Continued.

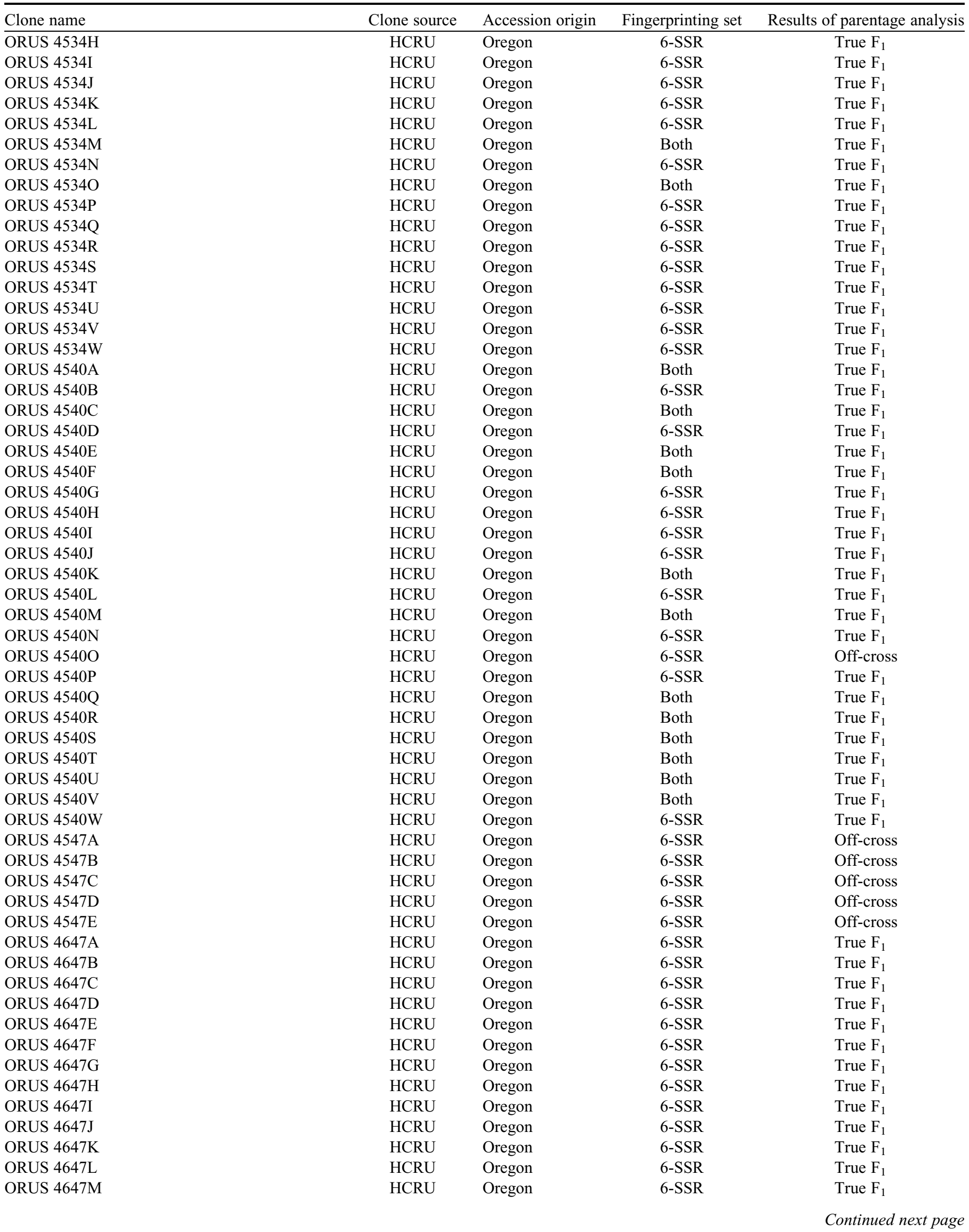


Supplemental Table 1. Continued.

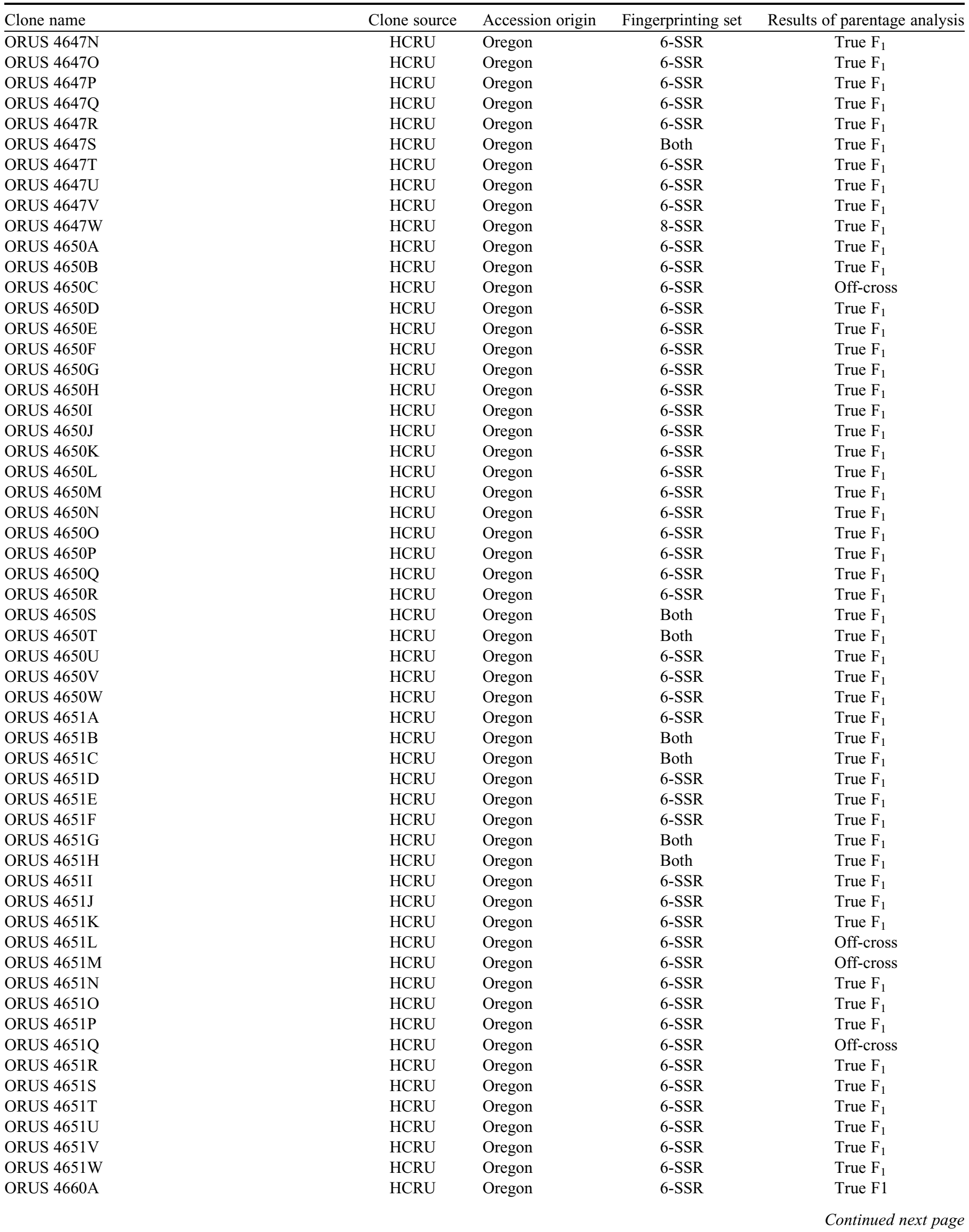


Supplemental Table 1. Continued.

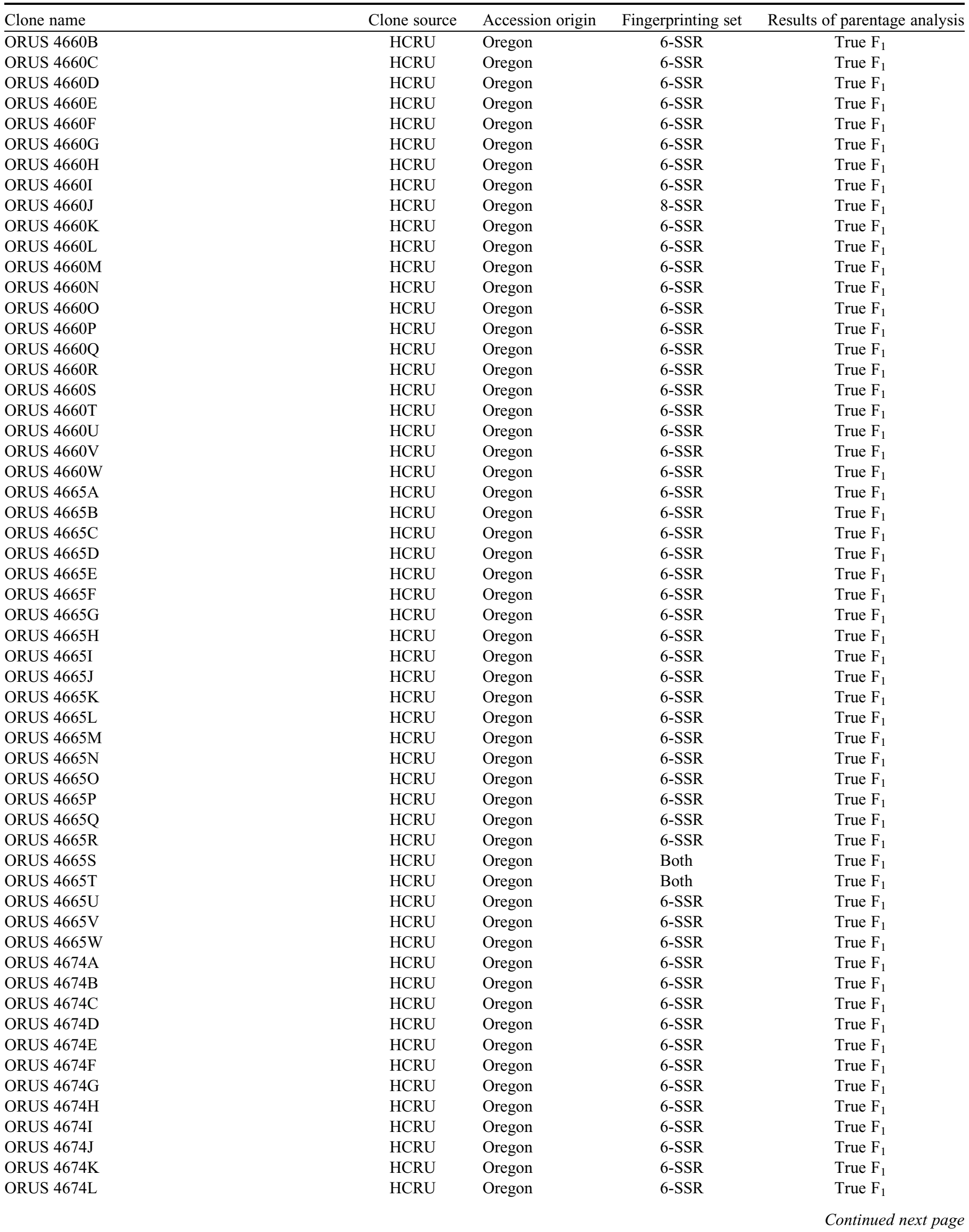


Supplemental Table 1. Continued.

\begin{tabular}{|c|c|c|c|c|}
\hline Clone name & Clone source & Accession origin & Fingerprinting set & Results of parentage analysis \\
\hline ORUS 4674M & HCRU & Oregon & 6-SSR & True $\mathrm{F}_{1}$ \\
\hline ORUS $4674 \mathrm{~N}$ & HCRU & Oregon & 6-SSR & True $F_{1}$ \\
\hline ORUS 46740 & HCRU & Oregon & 6-SSR & True $\mathrm{F}_{1}$ \\
\hline ORUS 4674Q & HCRU & Oregon & 6-SSR & True $\mathrm{F}_{1}$ \\
\hline ORUS 4674R & HCRU & Oregon & Both & True $\mathrm{F}_{1}$ \\
\hline ORUS 4754C & HCRU & Oregon & 6-SSR & True $\mathrm{F}_{1}$ \\
\hline ORUS 4754D & HCRU & Oregon & 6-SSR & True $\mathrm{F}_{1}$ \\
\hline ORUS 4754E & HCRU & Oregon & 6-SSR & True $\mathrm{F}_{1}$ \\
\hline ORUS 4754F & HCRU & Oregon & 6-SSR & True $\mathrm{F}_{1}$ \\
\hline ORUS 4754G & HCRU & Oregon & 6-SSR & True $\mathrm{F}_{1}$ \\
\hline ORUS $4754 \mathrm{H}$ & HCRU & Oregon & 6-SSR & True $\mathrm{F}_{1}$ \\
\hline ORUS $4754 \mathrm{M}$ & HCRU & Oregon & 6-SSR & True $\mathrm{F}_{1}$ \\
\hline ORUS $4754 \mathrm{~N}$ & HCRU & Oregon & 6-SSR & True $\mathrm{F}_{1}$ \\
\hline ORUS 47540 & HCRU & Oregon & 6-SSR & True $\mathrm{F}_{1}$ \\
\hline ORUS 4754P & HCRU & Oregon & 6-SSR & True $\mathrm{F}_{1}$ \\
\hline ORUS 4754Q & HCRU & Oregon & 6-SSR & True $\mathrm{F}_{1}$ \\
\hline ORUS 4772A & HCRU & Oregon & 6-SSR & True $\mathrm{F}_{1}$ \\
\hline ORUS 4772B & HCRU & Oregon & 6-SSR & True $\mathrm{F}_{1}$ \\
\hline ORUS 4772C & HCRU & Oregon & 6-SSR & True $\mathrm{F}_{1}$ \\
\hline ORUS 4772D & HCRU & Oregon & 6-SSR & True $\mathrm{F}_{1}$ \\
\hline ORUS 4772E & HCRU & Oregon & 6-SSR & True $\mathrm{F}_{1}$ \\
\hline ORUS 4772F & HCRU & Oregon & 6-SSR & True $\mathrm{F}_{1}$ \\
\hline ORUS $4772 \mathrm{~N}$ & HCRU & Oregon & 6-SSR & True $\mathrm{F}_{1}$ \\
\hline ORUS 47720 & HCRU & Oregon & 6-SSR & True $\mathrm{F}_{1}$ \\
\hline ORUS 4772P & HCRU & Oregon & 6-SSR & True $\mathrm{F}_{1}$ \\
\hline ORUS 4772Q & HCRU & Oregon & 6-SSR & True $\mathrm{F}_{1}$ \\
\hline ORUS 4772R & HCRU & Oregon & 6-SSR & True $\mathrm{F}_{1}$ \\
\hline ORUS 4773A & HCRU & Oregon & 6-SSR & True $\mathrm{F}_{1}$ \\
\hline ORUS 4773B & HCRU & Oregon & 6-SSR & True $\mathrm{F}_{1}$ \\
\hline ORUS 4773C & HCRU & Oregon & 6-SSR & True $\mathrm{F}_{1}$ \\
\hline ORUS 4773D & HCRU & Oregon & 6-SSR & True $\mathrm{F}_{1}$ \\
\hline ORUS 4773E & HCRU & Oregon & 6-SSR & True $\mathrm{F}_{1}$ \\
\hline ORUS 4773F & HCRU & Oregon & 6-SSR & True $\mathrm{F}_{1}$ \\
\hline Osage & UA & Arkansas & Both & \\
\hline Ouachita & HCRU & Arkansas & 6-SSR & \\
\hline Pacific CRUB 1220.001 & NCGR & Oregon & 8-SSR & \\
\hline Phenomenal CRUB 1817.001 & NCGR & California & 8-SSR & \\
\hline Prime-Ark $^{\circledR} 45$ & HCRU & Arkansas & Both & \\
\hline Prime-Ark ${ }^{\circledR} 45$ & UA & Arkansas & Both & \\
\hline Prime-Ark ${ }^{\circledR}$ Traveler & UA & Arkansas & Both & \\
\hline Prime-Jim & UA & Arkansas & 8-SSR & \\
\hline R. allegheniensis eastern upright CRUB 1781.001 & NCGR & Arkansas & 8-SSR & \\
\hline R. allegheniensis Pennsylvania CRUB 1152.001 & NCGR & Pennsylvania & 8-SSR & \\
\hline
\end{tabular}

Continued next page 
Supplemental Table 1. Continued.

\begin{tabular}{|c|c|c|c|c|}
\hline Clone name & Clone source & Accession origin & Fingerprinting set & Results of parentage analysis \\
\hline R. allegheniensis rual050 CRUB 2098.001 & NCGR & Southeast USA & 8-SSR & \\
\hline R. argutus NC 95-2-1 CRUB 1818.001 & NCGR & South Carolina & 8-SSR & \\
\hline R. caesius CRUB 149.003 & NCGR & Spain & 8-SSR & \\
\hline R. canadensis Georgia CRUB 817.001 & NCGR & Georgia (USA) & 8-SSR & \\
\hline R. canadensis NC 86-33-4 CRUB 785.001 & NCGR & North Carolina & 8-SSR & \\
\hline R. pensilvanicus BWP CRUB 2606.001 & NCGR & California & 8-SSR & \\
\hline R. sanctus 880334 CRUB 1054.002 & NCGR & Pakistan & 8-SSR & \\
\hline R. trivialis M-26826 CRUB 260.001 & NCGR & Georgia (USA) & 8-SSR & \\
\hline R. ulmifolius Turkey CRUB 23.001 & NCGR & Turkey & 8-SSR & \\
\hline R. ulmifolius Yugo CRUB 34.001 & NCGR & Montenegro & 8-SSR & \\
\hline R. ursinus CRUB611.001 & NCGR & Idaho & 8-SSR & \\
\hline Thornless Evergreen CRUB 991.001 & NCGR & Oregon & 8-SSR & \\
\hline Triple Crown CRUB 1946.001 & NCGR & Maryland & 8-SSR & \\
\hline Tupy CRUB 2192.001 & NCGR & Brazil & 8-SSR & \\
\hline Waldo CRUB 983.001 & NCGR & Oregon & 8-SSR & \\
\hline Whitford Thornless CRUB 722.002 & NCGR & Illinois & 8-SSR & \\
\hline Wild Treasure CRUB 2237.001 & NCGR & Oregon & 8-SSR & \\
\hline Young CRUB 131.001 & NCGR & Louisiana & 8-SSR & \\
\hline Young Thornless CRUB 2003.001 & NCGR & Louisiana & 8-SSR & \\
\hline
\end{tabular}


Supplemental Table 2. R code used to construct phylogenetic trees in the package "Poppr" (Kamvar et al., 2014, 2015) using a Bruvo's infinite allele, allele-sharing model (Bruvo et al., 2004; Metzger et al., 2016).

\# This block of code calls the packages 'poppr' and 'ape'

library(poppr)

library(ape)

\# This block of code imports the data from a text file. Data is imported from a .txt file containing \# the accessions, ploidy, and markers arranged in columns. Allele calls for each marker is \# formatted such that each observed allele is separtated by a colon ":" (e.g., 143:146). If SNP data

\# is in use, recode the SNPs as number 1-5 where one number indicates a deletion and the

\# remaining numbers indicate the nucleotide. Missing data is coded as "NA".

Mydat_table $=$ read.table $($ choose.files () , header $=$ TRUE, stringsAsFactors $=$ FALSE $)$

Accession_Names = as.character(Mydat_table[,1]) \#Column 1 of input file

Ploidy $=$ as.integer(Mydat_table[,2]) \#Column 2 of input file

Allele_Dat $=$ Mydat_table[3:ncol(Mydat_table)] \#Column 3 to end of input file

Mydat_genind = df2genind(Allele_Dat, sep = ":", ind.names = Accession_Names, ploidy = Ploidy, NA.char = "NA")

Mydat_genind_recoded $=$ recode_polyploids(Mydat_genind, addzero $=$ TRUE)

\# This block of code performs the phylogenetic analysis for a Bruvo's infinite allele, allele

\# sharing model

ssr_replen $=$ rep $(0.0001$, length(Mydat_genind@all.names $))$

Mytree = bruvo.boot(Mydat_genind_recoded, replen $=$ ssr_replen, sample $=2000$, cutoff $=70$, tree $=$ "njs", showtree $=$ FALSE, add $=$ FALSE, loss $=$ FALSE)

\# This block of code interactively performs K-means clustering

Mydat_clusters $=$ find.clusters $($ Mydat_genind, scale $=$ FALSE $)$

\# This block of code plots the tree produced with K-means clusters overlayed as differnet colors

\# for each group. See plot.phylo help for all plotting options

clustcolor $=$ rainbow(length(unique(Mydat_clusters\$grp) $))$

plot.phylo(Mytree, cex $=0.5$, edge. width $=0.5$, font $=2$, label.offset $=0.00125$, show.node.label $=$ FALSE, type $=$ "phylogram", tip.color $=$ clustcolor[Mydat_clusters\$grp], no.margin = TRUE)

nodelabels(Mytree \$node.label, adj = c(1.2, -0.4), frame $=$ "n", cex $=0.25)$

legend(locator(), legend $=\mathrm{c}(1$ :length(clustcolor) $)$, fill $=$ clustcolor, cex $=0.5$, bty $=$ "n", ncol = 4, title = "K-means Clusters")

add. scale.bar $(\operatorname{lwd}=0.5$, cex $=0.5$, font $=2$, ask $=$ TRUE) 